\title{
Sugar-Sweetened Beverage Intake Trends in US Adolescents and Their Association with Insulin Resistance-Related Parameters
}

\author{
Andrew A. Bremer, ${ }^{1}$ Peggy Auinger, ${ }^{2}$ and Robert S. Byrd ${ }^{1}$ \\ ${ }^{1}$ Department of Pediatrics, University of California Davis School of Medicine, Sacramento, CA 95817, USA \\ ${ }^{2}$ Department of Neurology, University of Rochester School of Medicine and Dentistry, Rochester, NY 14642, USA
}

Correspondence should be addressed to Andrew A. Bremer, andrew.bremer@ucdmc.ucdavis.edu

Received 28 May 2009; Accepted 24 July 2009

Academic Editor: Maria Luz Fernandez

Copyright ( $\odot 2010$ Andrew A. Bremer et al. This is an open access article distributed under the Creative Commons Attribution License, which permits unrestricted use, distribution, and reproduction in any medium, provided the original work is properly cited.

The purpose of this study was to evaluate current sugar-sweetened beverage (SSB) consumption trends and their association with insulin resistance-related metabolic parameters and anthropometric measurements by performing a cross-sectional analysis of the NHANES data during the years 1988-1994 and 1999-2004. Main outcome measures included SSB consumption trends, a homeostasis model assessment of insulin resistance, blood pressure, waist circumference, body mass index, and fasting concentrations of total cholesterol, HDL-cholesterol, LDL-cholesterol, and triglycerides. Although overall SSB consumption has increased, our data suggest that this increase was primarily due to an increase in the amount of SSBs consumed by males in the high-SSB intake group alone. Multivariate linear regression analyses also showed that increased SSB consumption was independently associated with many adverse health parameters. Factors other than SSB consumption must therefore be contributing to the increasing prevalence of obesity and metabolic syndrome in the majority of US children.

\section{Introduction}

The increased consumption of sugar-sweetened beverages (SSBs) over the past two decades has been implicated in the increased incidence of obesity and metabolic syndrome (MetS), a group of conditions associated with insulin resistance, including hypertension, dyslipidemia, central adiposity, and impaired glucose metabolism [1-3]. Based on data from the 1994-1996 Continuing Survey of Food Intakes by Individuals [4], the mean sugar consumption in all foods and beverages by Americans in the early 1990s comprised $16 \%$ of their total daily energy intake. However, sugar intake from SSBs alone, which currently represent the largest single caloric food source in the US [5], now approaches or exceeds $15 \%$ of the daily caloric intake in several population groups, including adolescents $[6,7]$.

As in the adult population, the prevalence of obesity and MetS in the US pediatric population is increasing [8-15]. Although the reasons for this are unknown, the increased consumption of SSBs has been postulated to be a contributing factor [16-18]. Experimental studies support the hypothesis that SSBs may increase energy intake and induce weight gain via their reduced satiety response, the promotion of a positive energy balance by liquid calories relative to isoenergetic solid calories, and their dysregulation of energy homeostasis [2, 19-22]. Although not all studies support an association between SSB consumption and obesity [23, 24], SSB intake has nonetheless been associated with increased body weight, increased fat mass, dyslipidemia, and blood pressure [2, 25-29]. Furthermore, the odds of a pediatric patient becoming obese-and therefore at risk for developing MetS-is reportedly increased by $\sim 60 \%$ for each additional SSB serving per day [16]; thus, the observation that the average intake of SSBs in US children and adolescents is now estimated to be more than double the amount consumed in the 1970s [30-32] has tremendous public health implications.

In order to evaluate the current consumption trends of SSBs and the association of SSB intake with insulin resistance-associated metabolic parameters and anthropometric measurements in the US pediatric population, we reviewed the National Health and Nutrition Examination Survey (NHANES) and performed these analyses in each available time period. We report our findings using data 
from US adolescents aged 12-19 years from NHANES III (1988-1994), NHANES 1999-2000, NHANES 2001-2002, and NHANES 2003-2004.

\section{Methods}

2.1. Study Design and Population. The NHANES is conducted by the National Center for Health Statistics of the Centers for Disease Control and Prevention (CDC), and is designed to monitor the health and nutritional status of the US civilian, noninstitutionalized population. NHANES III covered the years 1988-1994, and can be divided into two phases (1988-1991 and 1991-1994). Since 1999, NHANES has been planned and conducted as continuous annual surveys, and data are released in 2-year periods. A nationally representative sample is selected every year using a stratified multistage probability cluster sample design [33]; oversampling Mexican Americans and black individuals, adolescents aged 12-19 years, persons aged 60 years and older, lowincome white individuals, and pregnant women permit more precise estimates for these groups. This report is based on data from NHANES III, NHANES 1999-2000, NHANES 2001-2002, and NHANES 2003-2004, as these were the most recently available NHANES that had released all the data needed for the inclusion criteria, exclusion criteria, and outcome variables. The NHANES protocol was approved by the National Center for Health Statistics institutional review board (IRB), and written informed consent was obtained from all participants 18 years of age and older; for adolescents younger than 18 years of age, written informed assent was obtained in addition to parent or guardian consent. This study was approved by the IRB at the University of California, Davis.

2.2. Subjects. The NHANES protocol consists of a home interview performed by a trained interviewer, followed by a visit to an examination center, where participants undergo physical examinations, provide blood and urine samples, and complete additional questionnaires. The details of the participant examinations and laboratory assessments are available on the NHANES website (www.cdc.gov/nchs/nhanes.htm). For our study, only data from participants aged 12 to 19 years were analyzed; individuals were excluded from analyses if they had not fasted for at least 8 hours, were pregnant, and/or used steroids, blood glucose regulators, insulin, other anti-diabetic agents, growth hormone, or sex hormones.

2.3. Measurements. The NHANES III Dietary Data Collection system and the United States Department of Agriculture (USDA) Survey Nutrient Database (SND) were used for dietary intake data Coding [34]. NHANES 1999-2001 utilized the University of Texas Food Intake Analysis System along with the SND for 1999-2000 coding and the USDA Food and Nutrient Database for Dietary Studies (FNDDS) for 2001 coding [35]. Survey Net, a computer assisted food coding and data management system developed by the USDA, and the FNDDS were used for NHANES 2002-2004 data $[36,37]$.
Outcome variables included glucose levels, insulin levels, a homeostasis model assessment of insulin resistance (HOMA-IR), total cholesterol (TC) levels, high-density lipoprotein cholesterol (HDL-C) levels, low-density lipoprotein cholesterol (LDL-C) levels, triglyceride (TG) levels, the TG/HDL-C ratio, systolic blood pressure (SBP), diastolic blood pressure (DBP), waist circumference (WC), and body mass index (BMI; calculated as weight in kilograms divided by height in meters squared) percentile for age and sex (per the National Center for Health Statistics references) [38]. Since fasting glucose and fasting insulin levels were not collected from the subjects in NHANES III, HOMAIR (fasting glucose $(\mathrm{mM} / \mathrm{L}) \times$ fasting insulin $(\mathrm{mU} / \mathrm{mL}) / 22.5)$ [39] values from individuals in these cohorts could not be calculated. The TG/HDL-C ratio was included as an outcome variable due to its use as a marker of cardiovascular risk [40]. Mean WC is presented as the least squares mean, controlling for age and sex.

2.4. Definitions. Sugar-sweetened beverage information was obtained through a 24-hour dietary recall interview. (In NHANES 2003-2004, the 24-hour recall was assessed on two separate days; the first day was an in-person interview comparable to the previous NHANES study periods' primary interview mode, whereas the second day was a telephone interview 3-10 days later. For consistency in the methodology of data collection among the study periods, only the first day of the NHANES 2003-2004 24-hour recall was used in our analyses.) Sugar-sweetened beverages were defined as caloric soft drinks, colas, sugar-sweetened fruit drinks, or other sugar-sweetened beverages; pure fruit juices and diet soft drinks were not included. Sugar-sweetened beverage intake in grams ( $g$ ) for each reported beverage was divided by $250 \mathrm{~g}$ (a serving equivalent; approximately 8 ounces [oz] or a cup of beverage) and summed for each adolescent. In each NHANES analyzed, low SSB intake was defined as the lowest quintile $(\leq 20$ th percentile) of the sum of the number of SSB serving equivalents a subject consumed per day; medium was defined as the 2nd-4th quintiles ( $>20$ th $-<80$ th percentile); high was defined as the highest quintile ( $\geq 80$ th percentile). Units of SSB intake are defined as the number of SSB serving equivalents per day. Physical activity information was obtained during the interview questionnaire. The amount of physical activity performed per day was determined by the sum of (the mean number of times a subject did activity per day $) \times($ the average duration of each time in minutes $) \times($ the metabolic equivalent [MET] score) [41-43]. Energy intake information was also obtained from the interview questionnaire to determine the subject's caloric intake per day (in kilocalories).

2.5. Statistical Analysis. Statistical analyses were performed with SUDAAN, version 9.0 (Research Triangle Institute, Research Triangle Park, NC) using techniques appropriate for the complex NHANES survey design. All of the analyses used the NHANES-provided sampling weights that were calculated to take into account unequal probabilities of selection resulting from the sample design, nonresponse, and 
TABLE 1: Characteristics of US adolescents aged 12-19 years: NHANES III (1988-1994) and complete NHANES 1999-2004 cohorts.

\begin{tabular}{lccccccc}
\hline & $\begin{array}{c}\text { NHANES III } \\
\text { Phase I }\end{array}$ & $\begin{array}{c}\text { NHANES III } \\
\text { Phase II }\end{array}$ & NHANES & NHANES & NHANES & NHANES III & NHANES \\
& $1988-1991$ & $1991-1994$ & $1999-2000$ & $2001-2002$ & $2003-2004$ & $1988-1994$ & $1999-2004$ \\
\hline Number of Participants $(n)$ & 1531 & 1703 & 2308 & 2417 & 2242 & 3234 & 6967 \\
Age in years (mean) & 15.7 & 15.2 & 15.4 & 15.5 & 15.4 & 15.4 & 15.5 \\
Sex (\%) & & & & & & & \\
Male & 50.9 & 50.6 & 51.5 & 50.6 & 51.1 & 50.7 & 51.1 \\
Female & 49.1 & 49.4 & 48.5 & 49.4 & 48.9 & 49.3 & 48.9 \\
Race/ethnicity (\%) & & & & & & & \\
Non-Hispanic white & 66.9 & 65.1 & 56.8 & 63.8 & 64.9 & 66.0 & 62.1 \\
Non-Hispanic black & 15.4 & 15.6 & 14.9 & 13.9 & 15.7 & 15.5 & 14.8 \\
Mexican American & 8.2 & 8.3 & 13.0 & 9.0 & 11.1 & 8.3 & 10.9 \\
Other race-including multiracial & 4.8 & 4.8 & 7.3 & 5.3 & 3.6 & 4.8 & 5.3 \\
Other Hispanic & 4.7 & 6.2 & 8.0 & 8.0 & 4.7 & 5.4 & 6.9 \\
\hline
\end{tabular}

planned oversampling of selected subgroups, so that results are representative of the US community-dwelling population. Dietary variables were analyzed both as continuous variables and in quintiles to minimize the chance that a small number of extreme observations would have undue influence on the results. The data are presented as NHANES III (1988-1994) versus NHANES 1999-2004, both in their individual components (i.e., NHANES III, Phase I 19881991; NHANES III, Phase II 1991-1994; NHANES 19992000; NHANES 2001-2002; and NHANES 2003-2004) as well as in their entirety (NHANES 1988-1994 and NHANES 1999-2004) since both trend and aggregate analyses were performed. Descriptive statistics summarize the data and are expressed as the mean \pm the standard error (SE). Mean differences in outcomes comparing 1988-1994 to 1999-2004 in aggregate were tested for significance using $t$-tests. To test for linear trends, an ordinal variable representing the 5 time periods was included as a continuous dependent variable in regression analyses; each phase of NHANES III and each of the subsequent 2 year NHANES surveys were weighted to represent the US population [44]. Multivariate linear regression analyses were performed to determine independent associations between each outcome variable and the number of serving equivalents of SSBs consumed per day after adjusting for the amount of physical activity performed per day, age, sex, race, and energy intake per day (in kilocalories). All $P$ values are 2-sided and statistical significance was established a priori at $\alpha=.05$.

\section{Results}

3.1. Characteristics. The characteristics of the study participants are shown in Table 1. For NHANES III (1988-1994), a total of 3234 adolescents were studied; for NHANES 19992004, a total of 6967 adolescents were studied. The mean age, male/female ratio, and ethnic distributions between the study cohorts were comparable.

3.2. Trends in SSB Intake and Subgroup Analyses. The overall number of SSB serving equivalents consumed per day by each NHANES study period as well as the number of SSB serving equivalents consumed per day by the low-, medium-, and high-SSB intake groups from each cohort is shown in Table 2. A significant increase in overall SSB consumption over the 16 year time period was observed ( $P=.04$ for trend analysis; $P=.04$ for aggregate analysis). However, in subgroup analyses, no significant differences were observed in the amount of SSB consumption in the low- and medium-SSB intake populations among the study cohorts. Although no significant difference was noted in the trends of SSB consumption in the high-SSB intake populations among the study cohorts, a significant difference was observed in the high-SSB intake groups when the NHANES III versus NHANES 1999-2004 data were analyzed in aggregate $(P=.01)$. The high-SSB intake group in NHANES III consumed a mean of 6.6 SSB serving equivalents $(\sim 53 \mathrm{oz})$ per day, whereas the high-SSB intake group in NHANES 1999-2004 consumed a mean of 7.1 SSB serving equivalents $(\sim 57 \mathrm{oz})$ per day. For comparison, overall mean SSB consumption for the entire NHANES III and NHANES 1999-2004 cohorts were 2.8 SSB serving equivalents ( $\sim 22 \mathrm{oz})$ per day and 3.0 SSB serving equivalents $(\sim 24 \mathrm{oz})$ per day, respectively. Thus, in each cohort, the high-SSB intake group was consuming over twice the overall mean number of SSB serving equivalents per day.

3.3. Sex-Specific Subgroup Analyses. Since the number of SSBs consumed per day may be different between males and females, we also performed sex-specific subgroup analyses; these results are shown in Table 3 . In males, the pattern of overall SSB consumption trends and the significant differences observed between the high-SSB intake groups of NHANES III versus NHANES 1999-2004 mirror those found when studying the entire cohort. Specifically, a significant increase in male overall SSB consumption trends over the 16 year time period was observed $(P=.048$ for trend analysis); although the differences in male overall SSB consumption between NHANES III versus NHANES 1999-2004 did not reach statistical significance when the data were analyzed in aggregate, the analysis suggested a 
TABLE 2: Trends in SSB intake among adolescents aged 12-19 years: NHANES III (1988-1994), NHANES 1999-2000, NHANES 2001-2002, and NHANES 2003-2004. Low SSB intake defined as lowest quintile based on NHANES III cutoffs; medium defined as 2nd-4th quintiles; high defined as highest quintile. Mean indicates mean number of serving equivalents of sugar-sweetened beverages reported during 24 hour dietary recall. Significant results are in bold. Abbreviations: SSB, sugar-sweetened beverage; SE, standard error.

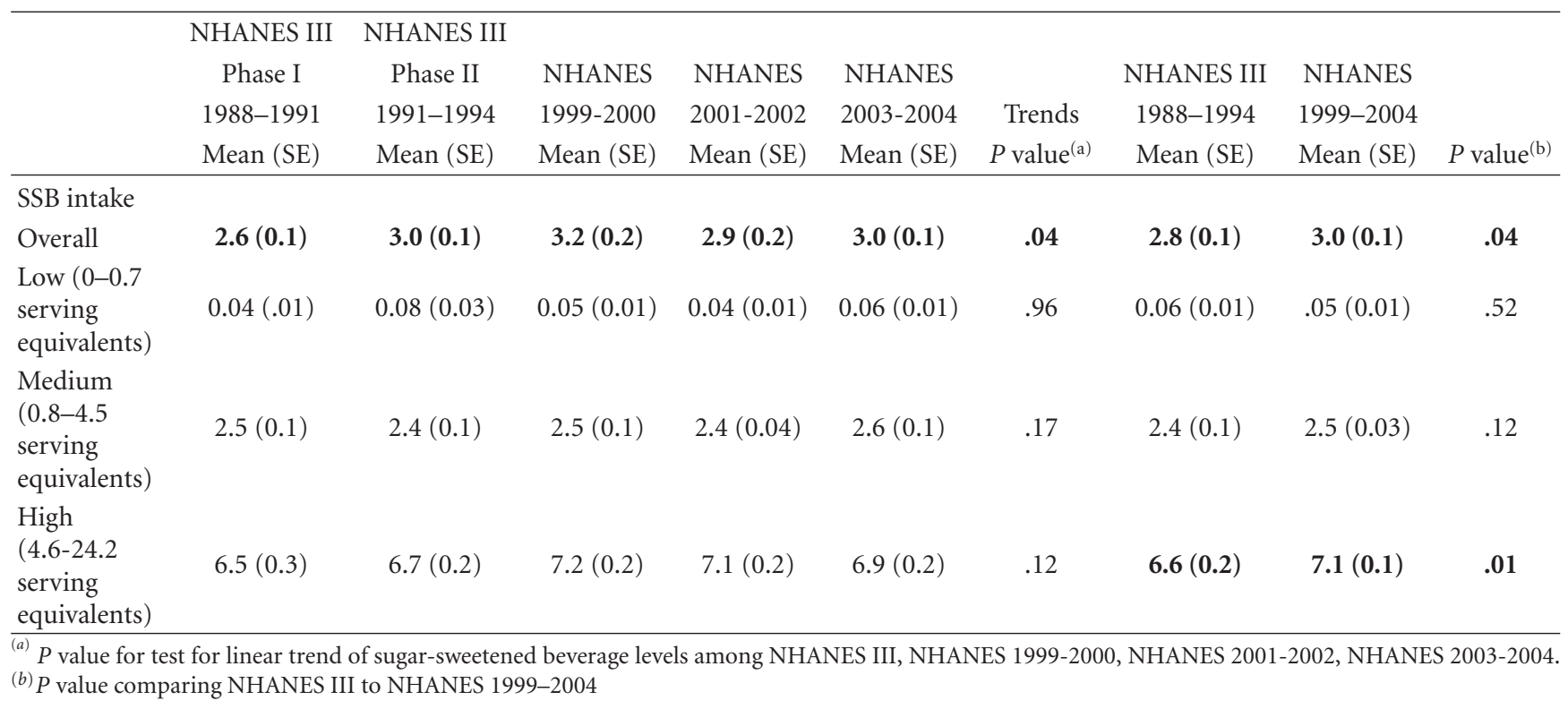

trend towards significance $(P=.051)$. Moreover, as in the overall cohort analyses, a significant difference was observed in the male high-SSB intake groups when the NHANES III versus NHANES 1999-2004 data were analyzed in aggregate $(P=.03)$. The male high-SSB intake group in NHANES III consumed a mean of $6.8 \mathrm{SSB}$ serving equivalents $(\sim$ $54 \mathrm{oz})$ per day, whereas the male high-SSB intake group in NHANES 1999-2004 consumed a mean of 7.3 SSB serving equivalents $(\sim 58 \mathrm{oz})$ per day. For comparison, overall mean male SSB consumption for the NHANES III and NHANES 1999-2004 cohorts were 3.3 SSB serving equivalents $(\sim$ $26 \mathrm{oz})$ per day and $3.6 \mathrm{SSB}$ serving equivalents $(\sim 29 \mathrm{oz})$ per day, respectively. Thus, overall mean SSB consumption was higher in males than in the overall cohort, especially in the high-SSB intake groups. In females, however, no significant changes were observed in either overall SSB consumption or the amount of SSB consumption in the low-, medium-, and high-SSB intake groups among the study cohorts. Moreover, SSB consumption by females (both overall and in each subgroup) in each study period was consistently less than that observed for the entire cohort.

3.4. Metabolic Parameters and Anthropometric Measurements Associated with SSB Intake. The multivariate linear regression analyses evaluating the relationship between SSB intake and insulin resistance-associated metabolic parameters and anthropometric measurements are shown in Table 4. All analyses were adjusted for the amount of physical activity performed per day, age, sex, race, and energy intake per day (in kilocalories). In the NHANES III cohort, each additional SSB serving equivalent consumed per day was associated with a $0.42 \mathrm{mg} / \mathrm{dL}$ decrease in HDL-C concentrations; HOMA-IR could not be assessed since fasting glucose and insulin concentrations were not obtained in the NHANES III study cohort. In the NHANES 1999-2004 cohort, each additional SSB serving equivalent consumed per day was associated with a $6 \%$ increase in HOMA-IR, a $0.16 \mathrm{mmHg}$ increase in SBP, a $0.47 \mathrm{~cm}$ increase in $\mathrm{WC}$, a 0.93 percentile increase in BMI, and a $0.48 \mathrm{mg} / \mathrm{dL}$ decrease in HDL-C concentrations.

\section{Discussion}

As has been reported elsewhere [7], we found a significant increase in the overall mean amount of SSB consumption over the past 16 years in these nationally representative samples of US adolescents aged 12-19 years. Specifically, compared to adolescents from the NHANES III study cohort, adolescents from the NHANES 1999-2004 study cohort consumed approximately $7 \%$ more SSB serving equivalents per day. However, our data suggest that this increase was primarily due to an increase in the amount of SSBs consumed by males in the high-SSB intake group alone. Furthermore, multivariate linear regression analyses showed that increased SSB consumption was independently associated with increased HOMA-IR, SBP, WC, and BMI percentile for age and sex and decreased HDL-C concentrations, reinforcing the negative impact that SSB consumption has on health parameters.

Given the strong association between SSB intake and altered metabolism [2, 25-29], our results are surprising. At the outset, we expected to find that SSB consumption had increased comparably among all consumption tiers of SSB intake and in both sexes, paralleling the increasing prevalence of obesity and MetS in the adolescent population [8-15]. However, our data suggest that the increase in SSB 
TABLE 3: Trends in SSB Intake among adolescents aged 12-19 years by sex: NHANES III (1988-1994), NHANES 1999-2000, NHANES 2001-2002, and NHANES 2003-2004. Low SSB intake defined as lowest quintile based on NHANES III cutoffs; medium defined as 2nd-4th quintiles; high defined as highest quintile. Mean indicates mean number of serving equivalents of sugar-sweetened beverages reported during 24 hour dietary recall. Significant results are in bold. Abbreviations: SSB, sugar-sweetened beverage; SE, standard error.

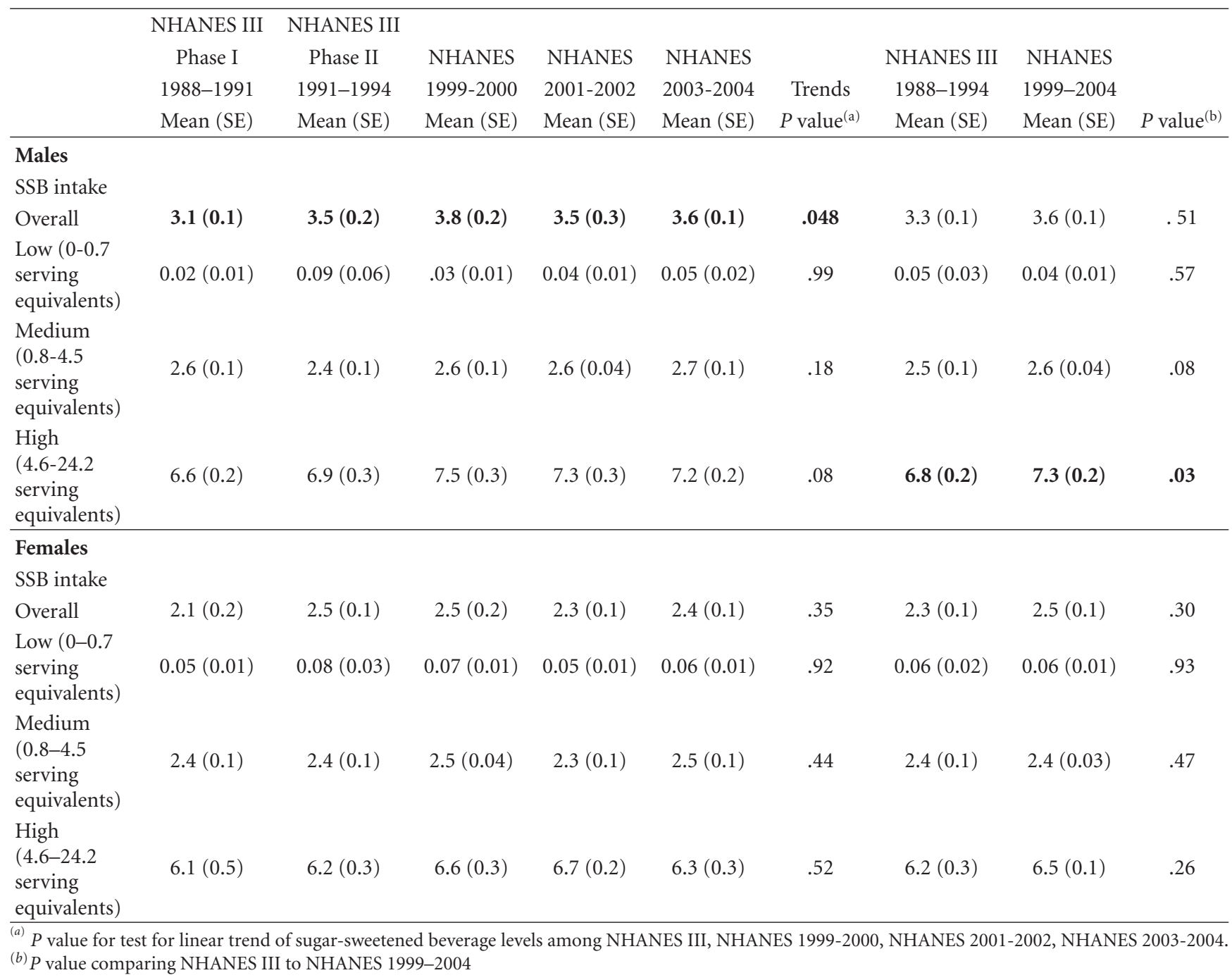

consumption that has been noted over the past 16 years in adolescents [7] is not universal, but rather confined to the top quintile of male SSB consumers, and that SSB consumption in most adolescents (the bottom four quintiles in males and all quintiles in females) has not increased over this time period. Thus, as has been reported elsewhere $[45,46]$, factors other than SSB consumption in the pediatric population—such as an increase in total caloric intake, a decrease in calcium and other nutrient intake, a decrease in daily physical activity, and potential genetic influencesmust also be contributing to the increasing prevalence of pediatric obesity and MetS.

Nevertheless, as shown by our linear regression analyses, SSB consumption is associated with adverse metabolic parameters [16-18]. It is thus reassuring that legislative and regulatory actions have specifically targeted SSB consumption in schools as one means to promote improved adolescent health [47]. Initiatives such as the one in 2006 between major US beverage manufacturers, the American Heart Association, and the Clinton Foundation to establish voluntary guidelines regarding the type of beverages sold at schools have brought national attention to the negative metabolic effects associated with SSB consumption, and although some reports suggest that initiatives to restrict SSB sales in schools may have only a marginal impact on overall SSB consumption [7] they are most likely at least in part responsible for the recent finding that the prevalence of high BMI among US children and adolescents between 2003-2004 and 2005-2006 has not changed [48]. Previous research has also shown a beneficial effect on body weight by reducing SSB consumption in those adolescents with an elevated BMI, supporting the current American Academy of Pediatrics guidelines to limit SSB consumption [49].

Moreover, our data show that SSB intake in the top quintile of SSB consumers from each of the NHANES 19992004 study periods has begun to decline (7.2 SSB serving 
TABLE 4: Regression analyses of metabolic parameters and anthropometric Measurements associated with SSB intake among US adolescents aged 12-19 years: NHANES III (1988-1994), NHANES 1999-2000, NHANES 2001-2002, NHANES 2003-2004, and complete NHANES 1999-2004 cohorts. Beta (SE) represents the number of SSBs consumed per day, adjusting for amount of physical activity performed per day, age, sex, ethnicity, and energy intake per day (in kilocalories). Abbreviations: HOMA-IR, homeostasis model assessment of insulin resistance; TC, total cholesterol; TG, triglyceride; HDL-C, high-density lipoprotein cholesterol; LDL-C, low-density lipoprotein cholesterol; SBP, systolic blood pressure; DBP, diastolic blood pressure; BMI, body mass index; WC, waist circumference; SE, standard error.

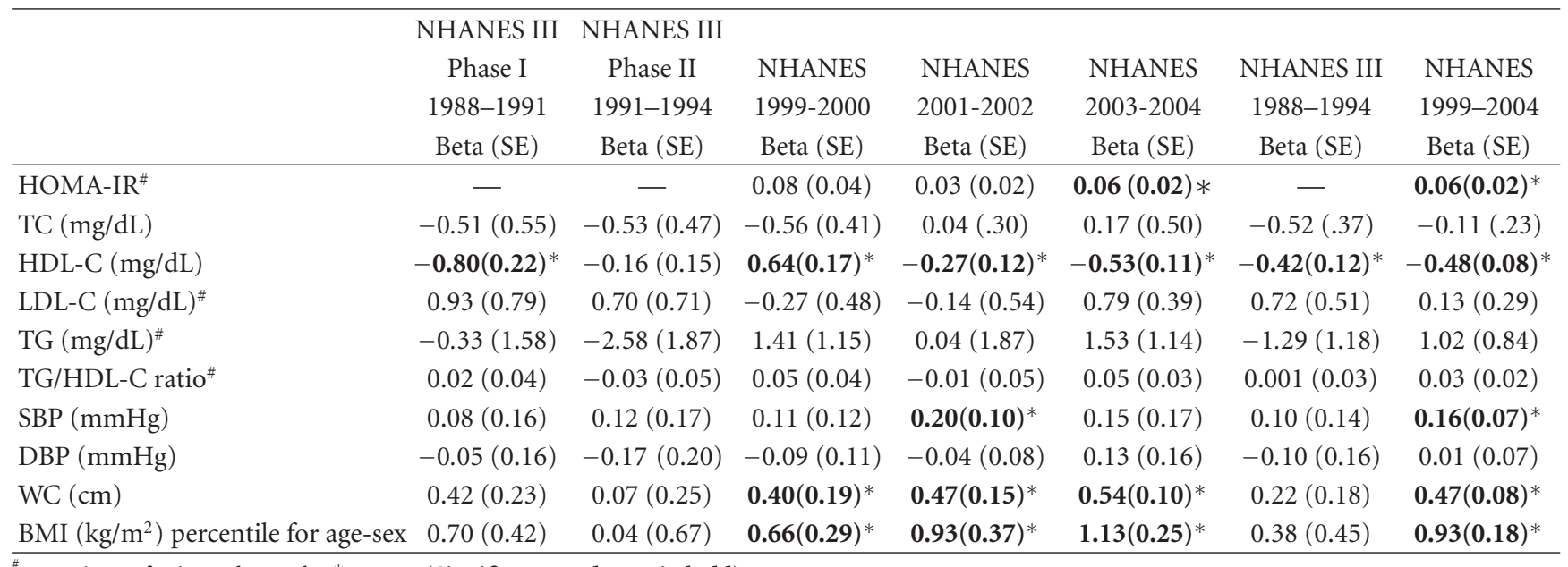

\# morning \& fasting subsample. ${ }^{*} P<.05$ (Significant results are in bold).

equivalents per day in 1999-2000, to 7.1 SSB serving equivalents per day in 2001-2002, to 6.9 SSB serving equivalents per day in 2003-2004) after increasing dramatically from the amount of SSB consumption in NHANES III (6.5 SSB serving equivalents per day in 1988-1991, and 6.7 SSB serving equivalents per day in 1991-1994). Whether this trend continues, and whether it is associated with changes in the prevalence of pediatric obesity and MetS, warrants further investigation.

One limitation to this study is that the data are crosssectional, and thus cannot infer causality; another is that the analyses are confined to adolescents aged $12-19$ years. We can also not exclude the possibility that significant differences in either the age at which children begin consuming SSBs and/or the amounts of SSBs consumed among children in the pre-teen years increase the rate of obesity and MetS in adolescents, as both of these scenarios would not be captured by our analyses. Moreover, since the pubertal status of subjects participating in NHANES has not been documented since NHANES III, we are unable to longitudinally adjust for the subjects' degree of pubertal maturation. Studies such as ours that utilize questionnaires also have other inherent limitations: (i) the recall method is subject to inaccuracy and bias, especially with behaviors such as dietary habits [50] and levels of exercise [41], (ii) individual's dietary habits and levels of exercise can vary greatly from one day to the next, limiting the reliability of short-term recall on longterm patterns. However, given that overweight subjects often underreport their levels of energy intake [50] and less active adolescents often overestimate their degree of physical fitness [41], we can have confidence in our results since these biases would be expected to diminish rather than enhance our ability to find significant associations between SSB consumption and insulin resistance-associated measures.

\section{Conclusions}

Although the mean overall amount of SSB consumption in US adolescents has increased over the past 16 years, paralleling the increase in pediatric obesity and MetS, our data suggest that this observation is primarily due to increased SSB intake in the top quintile of male SSB consumers. Thus, increased consumption of SSBs is not solely responsible for the increasing prevalence of obesity and MetS for the majority of US adolescents. Public health efforts aimed at addressing other factors linked with adverse metabolic parameters and anthropometric measurements, such as decreasing physical activity and increasing consumption of fast foods and other calorie-dense and nutritionallypoor foods, are therefore needed to adequately address the ongoing epidemic of pediatric obesity and MetS.

\section{Acknowledgments}

The authors thank Dr. Daphne Carlson Bremer and Dr. Daniel Tancredi for their assistance in the preparation of this manuscript. All authors had full access to the data in the study and take responsibility for the integrity of the data and the accuracy of the data analysis. This work was supported by Grant nos. KL2 RR024144 and UL1 RR024146 from the National Center for Research Resources (NCRR), a component of the National Institutes of Health (NIH), and NIH Roadmap for Medical Research. Its contents are solely the responsibility of the authors and do not necessarily represent the official view of NCRR or NIH. Information on NCRR is available at http://www.ncrr.nih.gov. Information on Re-engineering the Clinical Research Enterprise can be obtained from http://nihroadmap.nih.gov/clinicalresearch /overview-translational.asp. 


\section{References}

[1] G. A. Bray, S. J. Nielsen, and B. M. Popkin, "Consumption of high-fructose corn syrup in beverages may play a role in the epidemic of obesity," American Journal of Clinical Nutrition, vol. 79, no. 4, pp. 537-543, 2004.

[2] P. J. Havel, "Dietary fructose: implications for dysregulation of energy homeostasis and lipid/carbohydrate metabolism," Nutrition Reviews, vol. 63, no. 5, pp. 133-157, 2005.

[3] S. N. Bleich, Y. C. Wang, Y. Wang, and S. L. Gortmaker, "Increasing consumption of sugar-sweetened beverages among US adults: 1988-1994 to 1999-2004," American Journal of Clinical Nutrition, vol. 89, no. 1, pp. 372-381, 2009.

[4] J. F. Guthrie and J. F. Morton, "Food sources of added sweeteners in the diets of Americans," Journal of the American Dietetic Association, vol. 100, no. 1, pp. 43-51, 2000.

[5] G. Block, "Foods contributing to energy intake in the US: data from NHANES III and NHANES 1999-2000," Journal of Food Composition and Analysis, vol. 17, no. 3-4, pp. 439-447, 2004.

[6] D. S. West, Z. Bursac, D. Quimby, et al., "Self-reported sugarsweetened beverage intake among college students," Obesity, vol. 14, no. 10, pp. 1825-1831, 2006.

[7] Y. C. Wang, S. N. Bleich, and S. L. Gortmaker, "Increasing caloric contribution from sugar- sweetened beverages and $100 \%$ fruit juices among US children and adolescents, 1988 2004," Pediatrics, vol. 121, no. 6, pp. e1604-e1614, 2008.

[8] G. E. Duncan, S. M. Li, and X.-H. Zhou, "Prevalence and trends of a metabolic syndrome phenotype among U.S. adolescents, 1999-2000," Diabetes Care, vol. 27, no. 10, pp. 2438-2443, 2004.

[9] R. Weiss, J. Dziura, T. S. Burgert, et al., "Obesity and the metabolic syndrome in children and adolescents," The New England Journal of Medicine, vol. 350, no. 23, pp. 2362-2374, 2004.

[10] S. Ten and N. Maclaren, "Insulin resistance syndrome in children," Journal of Clinical Endocrinology and Metabolism, vol. 89, no. 6, pp. 2526-2539, 2004.

[11] N. K. Maclaren, S. Gujral, S. Ten, and R. Motagheti, "Childhood obesity and insulin resistance," Cell Biochemistry and Biophysics, vol. 48, no. 2-3, pp. 73-78, 2007.

[12] S. D. De Ferranti and S. K. Osganian, "Epidemiology of paediatric metabolic syndrome and type 2 diabetes mellitus," Diabetes and Vascular Disease Research, vol. 4, no. 4, pp. 285296, 2007.

[13] J. M. Saland, "Update on the metabolic syndrome in children," Current Opinion in Pediatrics, vol. 19, no. 2, pp. 183-191, 2007.

[14] J. M. Lee, M. J. Okumura, M. M. Davis, W. H. Herman, and J. G. Gurney, "Prevalence and determinants of insulin resistance among U.S. adolescents: a population-based study," Diabetes Care, vol. 29, no. 11, pp. 2427-2432, 2006.

[15] S. Cook, P. Auinger, C. Li, and E. S. Ford, "Metabolic syndrome rates in United States Adolescents, from the National Health and Nutrition Examination Survey, 1999-2002," Journal of Pediatrics, vol. 152, no. 2, pp. 165-170, 2008.

[16] D. S. Ludwig, K. E. Peterson, and S. L. Gortmaker, "Relation between consumption of sugar-sweetened drinks and childhood obesity: a prospective, observational analysis," The Lancet, vol. 357, no. 9255, pp. 505-508, 2001.

[17] J. A. Welsh, M. E. Cogswell, S. Rogers, H. Rockett, Z. Mei, and L. M. Grummer-Strawn, "Overweight among low-income preschool children associated with the consumption of sweet drinks: Missouri, 1999-2002," Pediatrics, vol. 115, no. 2, pp. e223-e229, 2005.
[18] V. S. Malik, M. B. Schulze, and F. B. Hu, "Intake of sugarsweetened beverages and weight gain: a systematic review," American Journal of Clinical Nutrition, vol. 84, no. 2, pp. 274288, 2006.

[19] D. P. DiMeglio and R. D. Mattes, "Liquid versus solid carbohydrate: effects on food intake and body weight," International Journal of Obesity, vol. 24, no. 6, pp. 794-800, 2000.

[20] M.-P. St-Onge, F. Rubiano, W. F. DeNino, et al., "Added thermogenic and satiety effects of a mixed nutrient vs a sugaronly beverage," International Journal of Obesity, vol. 28, no. 2, pp. 248-253, 2004.

[21] K.-A. Le and L. Tappy, "Metabolic effects of fructose," Current Opinion in Clinical Nutrition and Metabolic Care, vol. 9, no. 4, pp. 469-475, 2006.

[22] A. C. Rutledge and K. Adeli, "Fructose and the metabolic syndrome: pathophysiology and molecular mechanisms," Nutrition Reviews, vol. 65, no. 6, pp. S13-S23, 2007.

[23] T. M. O'Connor, S.-J. Yang, and T. A. Nicklas, "Beverage intake among preschool children and its effect on weight status," Pediatrics, vol. 118, no. 4, pp. e1010-e1018, 2006.

[24] R. A. Forshee, P. A. Anderson, and M. L. Storey, "Sugarsweetened beverages and body mass index in children and adolescents: a meta-analysis," American Journal of Clinical Nutrition, vol. 87, no. 6, pp. 1662-1671, 2008.

[25] A. Raben, T. H. Vasilaras, A. C. Moller, and A. Astrup, "Sucrose compared with artificial sweeteners: different effects on ad libitum food intake and body weight after 10 wk of supplementation in overweight subjects," American Journal of Clinical Nutrition, vol. 76, no. 4, pp. 721-729, 2002.

[26] M. M. Swarbrick, K. L. Stanhope, S. S. Elliott, et al., "Consumption of fructose-sweetened beverages for 10 weeks increases postprandial triacylglycerol and apolipoprotein-B concentrations in overweight and obese women," British Journal of Nutrition, vol. 100, no. 5, pp. 947-952, 2008.

[27] K. L. Stanhope, S. C. Griffen, B. R. Bair, M. M. Swarbrick, N. L. Keim, and P. J. Havel, "Twenty-four-hour endocrine and metabolic profiles following consumption of high-fructose corn syrup-, sucrose-, fructose-, and glucose-sweetened beverages with meals," American Journal of Clinical Nutrition, vol. 87, no. 5, pp. 1194-1203, 2008.

[28] K. L. Stanhope and P. J. Havel, "Endocrine and metabolic effects of consuming beverages sweetened with fructose, glucose, sucrose, or high-fructose corn syrup," American Journal of Clinical Nutrition, vol. 88, no. 6, pp. 1733S-1737S, 2008.

[29] K. L. Stanhope, J. M. Schwarz, N. L. Keim, et al., "Consuming fructose-sweetened, not glucose-sweetened, beverages increases visceral adiposity and lipids and decreases insulin sensitivity in overweight/obese humans," Journal of Clinical Investigation, vol. 119, no. 5, pp. 1322-1334, 2009.

[30] C. Cavadini, A. M. Siega-Riz, and B. M. Popkin, "US adolescent food intake trends from 1965 to 1996," Archives of Disease in Childhood, vol. 83, no. 1, pp. 18-24, 2000.

[31] S. A. French, B.-H. Lin, and J. F. Guthrie, "National trends in soft drink consumption among children and adolescents age 6 to 17 years: prevalence, amounts, and sources, 1977/1978 to 1994/1998," Journal of the American Dietetic Association, vol. 103, no. 10, pp. 1326-1331, 2003.

[32] S. J. Nielsen and B. M. Popkin, "Changes in beverage intake between 1977 and 2001," American Journal of Preventive Medicine, vol. 27, no. 3, pp. 205-210, 2004.

[33] Centers for Disease Control and Prevention (CDC), "National Center for Health Statistics (NCHS). National Health and Nutrition Examination Survey Data," Department of Health 
and Human Services, Centers for Disease Control and Prevention, Hyattsville, Md, USA, 1999-2004, August 2007, http://www.cdc.gov/nchs/nhanes.htm.

[34] M. McDowell, R. Briefel, R. Warren, M. Buzzard, D. Seskanich, and S. Gardner, "The dietary data collection system: an automated interview and coding system for NHANES III," in Proceedings of the 14th National Nutrient Databank Conference, CBORD Group, Ithaca, NY, USA, 1990.

[35] University of Texas-Houston Health Science Center (2002), "Food Intake Analysis System," August 2007, http://www.uth .tmc.edu/otm/available-technologies/neds/Food-Intake-Analysis-System.html.

[36] Agricultural Research Service, Beltsville Human Nutrition Research Center, “Food Surveys Research Group," USDA Food and Nutrient Database for Dietary Studies, Beltsville, Md, USA, August 2007, http://www.ars.usda.gov/ba/bhnrc/fsrg.

[37] N. Raper, B. Perloff, L. Ingwersen, L. Steinfeldt, and J. Anand, "An overview of USDA's Dietary Intake Data system," Journal of Food Composition and Analysis, vol. 17, no. 3-4, pp. 545-555, 2004.

[38] Centers for Disease Control and Prevention (CDC), "CDC Growth Charts," National Center for Health Statistics (NCHS). Department of Health and Human Services, Centers for Disease Control and Prevention, Hyattsville, Md, USA, 1999-2004, August 2007, http://www.cdc.gov/growthcharts.

[39] D. R. Matthews, J. P. Hosker, and A. S. Rudenski, "Homeostasis model assessment: insulin resistance and $\beta$-cell function from fasting plasma glucose and insulin concentrations in man," Diabetologia, vol. 28, no. 7, pp. 412-419, 1985.

[40] Z. Quijada, M. Paoli, Y. Zerpa, et al., "The triglyceride/HDLcholesterol ratio as a marker of cardiovascular risk in obese children; association with traditional and emergent risk factors," Pediatric Diabetes, vol. 9, no. 5, pp. 464-471, 2008.

[41] R. R. Pate, C.-Y. Wang, M. Dowda, S. W. Farrell, and J. R. O'Neill, "Cardiorespiratory fitness levels among US youth 12 to 19 years of age: findings from the 1999-2002 national health and nutrition examination survey," Archives of Pediatrics and Adolescent Medicine, vol. 160, no. 10, pp. 1005-1012, 2006.

[42] W. L. Haskell, I.-M. Lee, R. R. Pate, et al., "Physical activity and public health: updated recommendation for adults from the American College of Sports Medicine and the American Heart Association," Circulation, vol. 116, no. 9, pp. 1081-1093, 2007.

[43] A. A. Bremer, P. Auinger, and R. S. Byrd, "Relationship between insulin resistance-associated metabolic parameters and anthropometric measurements with sugar-sweetened beverage intake and physical activity levels in US adolescents: findings from the 1999-2004 National Health and Nutrition Examination Survey," Archives of Pediatrics and Adolescent Medicine, vol. 163, no. 4, pp. 328-335, 2009.

[44] A. K. Kant and B. I. Graubard, "Secular trends in patterns of self-reported food consumption of adult Americans: NHANES 1971-1975 to NHANES 1999-2002," American Journal of Clinical Nutrition, vol. 84, no. 5, pp. 1215-1223, 2006.

[45] C. M. Bachman, T. Baranowski, and T. A. Nicklas, "Is there an association between sweetened beverages and adiposity?" Nutrition Reviews, vol. 64, no. 4, pp. 153-174, 2006.

[46] Y. Pan and C. A. Pratt, "Metabolic syndrome and its association with diet and physical activity in US adolescents," Journal of the American Dietetic Association, vol. 108, no. 2, pp. 276286, 2008.

[47] M. M. Mello, J. Pomeranz, and P. Moran, "The interplay of public health law and industry self-regulation: the case of sugar-sweetened beverage sales in schools," American Journal of Public Health, vol. 98, no. 4, pp. 595-604, 2008.

[48] C. L. Ogden, M. D. Carroll, and K. M. Flegal, "High body mass index for age among US children and adolescents, 2003-2006," Journal of the American Medical Association, vol. 299, no. 20, pp. 2401-2405, 2008.

[49] C. B. Ebbeling, H. A. Feldman, S. K. Osganian, V. R. Chomitz, S. J. Ellenbogen, and D. S. Ludwig, "Effects of decreasing sugar-sweetened beverage consumption on body weight in adolescents: a randomized, controlled pilot study," Pediatrics, vol. 117, no. 3, pp. 673-680, 2006.

[50] R. R. Briefel, C. T. Sempos, M. A. McDowell, S. Chien, and K. Alaimo, "Dietary methods research in the third National Health and Nutrition Examination Survey: underreporting of energy intake," American Journal of Clinical Nutrition, vol. 65, supplement 4, pp. 1203S-1209S, 1997. 


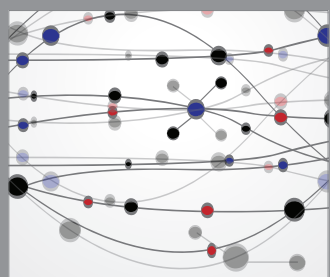

The Scientific World Journal
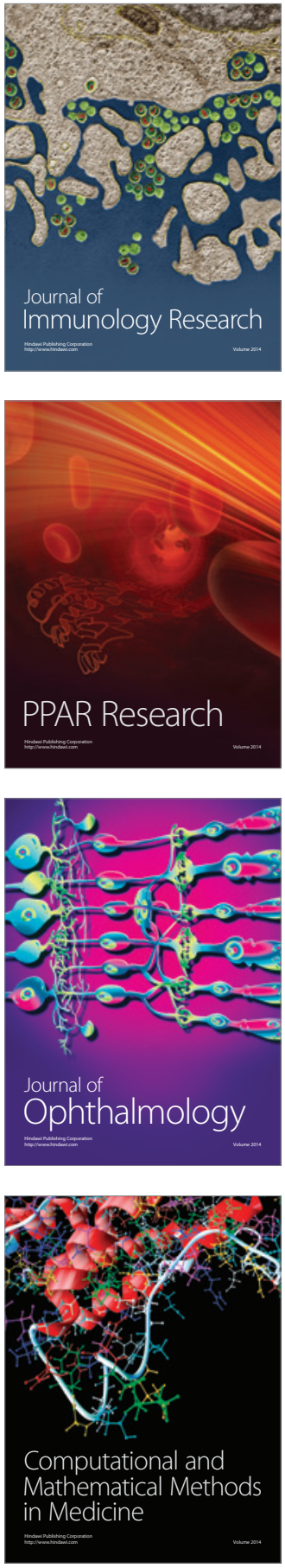

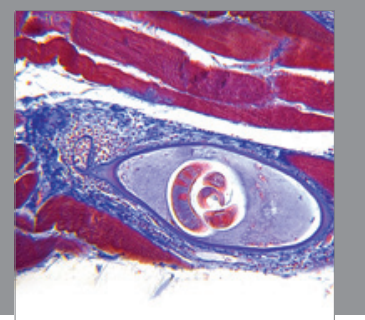

Gastroenterology

Research and Practice
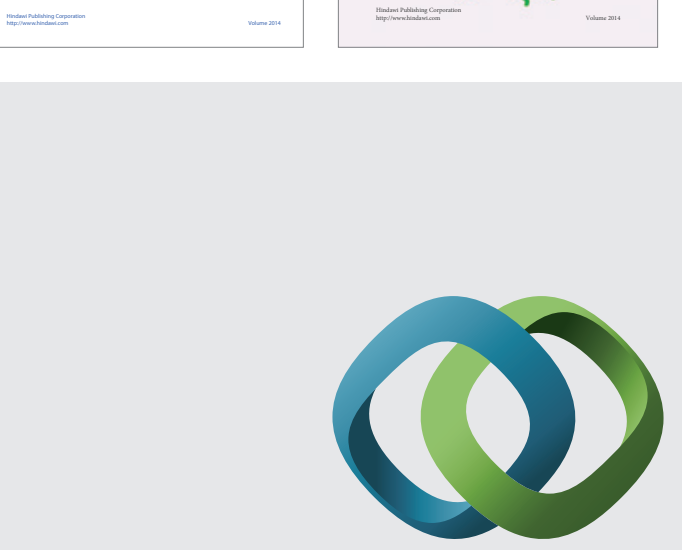

\section{Hindawi}

Submit your manuscripts at

http://www.hindawi.com
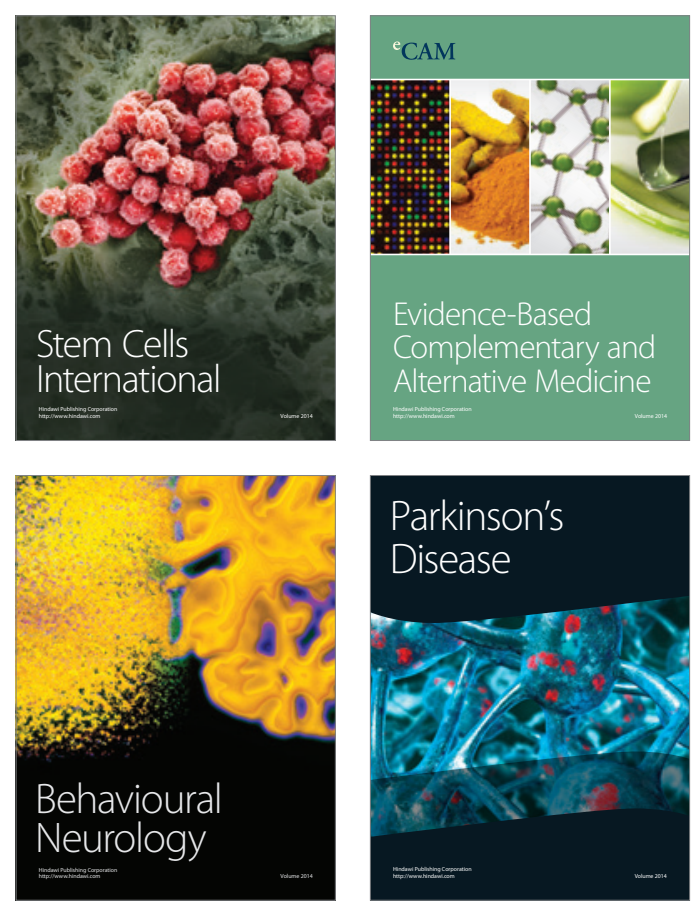

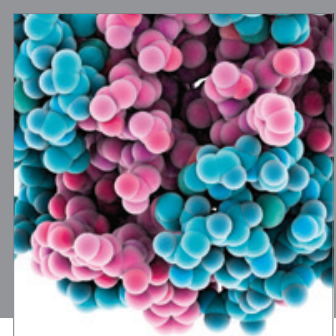

Journal of
Diabetes Research

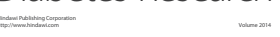

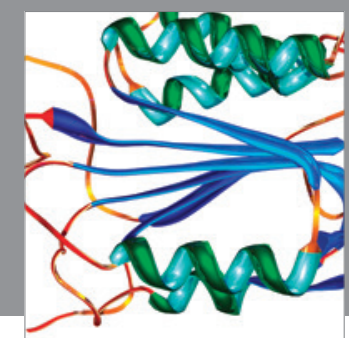

Disease Markers
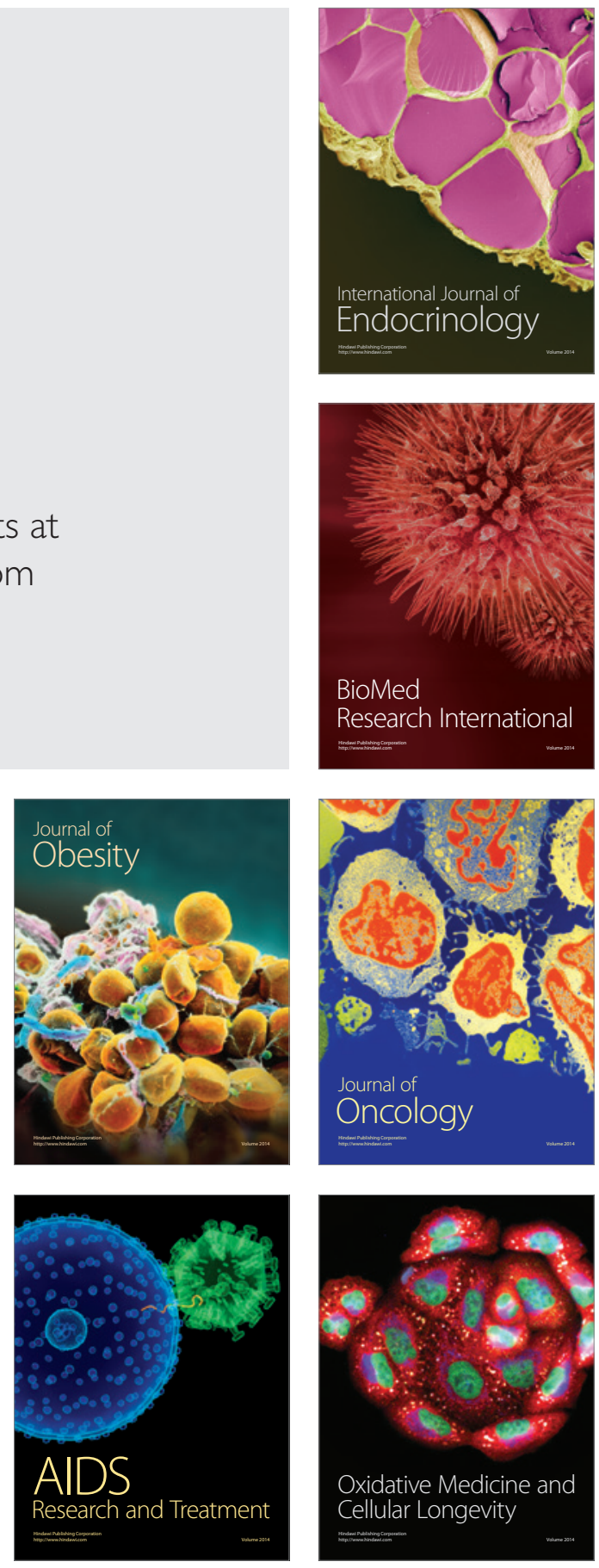